Mauro José Lahm

CARDOSO

Fabiano Sélos COSTA ${ }^{2}$

Lucy Marie Ribeiro MUNIZ ${ }^{3}$

Ana Cristina Aranha ZALITH

Carlos Roberto PADOVANI ${ }^{5}$

Flávio Ferrari ARAGON ${ }^{5}$

Correspondência Para:

MAURO JOSÉ LAHMCARDOSO

Hospital Veterinário

Faculdades Luiz Meneghel

Universidade Estadual do Paraná

BR 369, KM 54

Caixa Postal 261

80360-000 - Bandeirantes - PR

maurolahm@ffalm.br

Recebido para publicação: 18/05/2005 Aprovado para publicação: 07/02/2007

\title{
Mensuração do tamanho cardíaco em radiografias de gatos com hipertireoidismo experimental
}

\author{
1 - Professor de Clínica Médica e Semiologia de Pequenos Animais da \\ Faculdades Luiz Meneghel da Universidade Estadual do Paraná, \\ Bandeirantes - PR e Doutorando da Faculdade de Medicina Veterinária e \\ Zootecnia da Universidade Estadual Paulista, Botucatu - SP \\ 2 - Professor de Diagnóstico por Imagem e Clínica Médica de Animais de \\ Companhia da Universidade Federal do Espirito Santo, Alegre - ES e \\ Doutorando da Faculdade de Medicina Veterinária e Zootecnia da \\ Universidade Estadual Paulista, Botucatu - SP \\ 3 - Professor de Radiologia do Departamento de Reprodução Animal e \\ Radiologia Veterinária da Faculdade de Medicina Veterinária e Zootecnia da \\ Universidade Estadual Paulista, Botucatu - SP \\ 4 - Médica Veterinária e Doutorando da Faculdade de Medicina Veterinária \\ e Zootecnia da Universidade Estadual Paulista, Botucatu - SP \\ 5 - Departamento de Bioestatística do Instituto de Biologia da Faculdade de \\ Medicina Veterinária e Zootecnia da Universidade Estadual Paulista, \\ Botucatu - SP
}

\section{Resumo}

O hipertireoidismo felino ou tireotoxicose é a doença endócrina mais freqüente em gatos domésticos. O hipertireoidismo felino é uma desordem multissistêmica associada com aumento das concentrações dos hormônios tireoidianos circulantes, triidotironina (T3) e tiroxina (T4). Anormalidades cardiovasculares em gatos com hipertireoidismo espontâneo já foram bem descritas. O objetivo deste trabalho foi realizar a mensuração cardíaca em 19 gatos submetidos a tireotoxicose experimental, utilizando o "vertebral heart size" (VHS). A mensuração utilizando o VHS foi realizada a partir de radiografias torácicas lateral, dorsoventral e ventrodorsal. As radiografias torácicas laterais foram mais eficazes do que as ventrodorsal e dorsoventral em ilustrar o aumento progressivo do coração. O VHS é um método fácil de ser usado, permitindo a avaliação do tamanho cardíaco em gatos hipertireóideos, e facilita a identificação de cardiomegalia e progressão do tamanho cardíaco.

\section{Revisão de Literatura}

O hipertireoidismo ou tireotoxicose atualmente é considerada a endocrinopatia mais freqüente dos felinos ${ }^{1}$. O hipertireoidismo felino é uma alteração clínica multissistêmica resultante de excessivas concentrações dos hormônios tireoidianos (tiroxina e triiodotironina) $)^{2,3}$.

O hipertireoidismo provoca alterações no sistema cardiovascular, principalmente no coração ${ }^{4}$. No exame radiográfico de tórax, de gatos com hipertireoidismo, pode-se observar: cardiomegalia leve a grave, derrame pleural e edema pulmonar ${ }^{5,6,7}$.

As concentrações séricas elevadas da T3 total (TT3), T3 livre (LT3), T4 total (TT4) e T4 livre (LT4) são na maioria dos casos os principais indicativos de hipertireoidismo felino $^{2,8}$. Os valores normais de TT4 variam de 9,7 a $56,3 \mathrm{nmol} / \mathrm{L}$ ou 0,7 a $3,8 \mathrm{mg} / \mathrm{dL}$, para LT4 de 12 a $51 \mathrm{pmol} / \mathrm{L}$ ou 0,93 a 3,96 ng/dL e para TT3 de 0,16 a $1,9 \mathrm{nmol} / \mathrm{L}$ ou 10 a 104 $\mathrm{ng} / \mathrm{dL}$, conforme diversos autores ${ }^{3,4,5,9}$. Valores dos hormônios tireoidianos de gatos com 
hipertireoidismo espontâneo variam de 35,9 a $693,6 \mathrm{nmol} / \mathrm{L}$ ou 2,8 a $26,8 \mathrm{mg} / \mathrm{dL}$ para a TT4, de 41 a $144 \mathrm{pmol} / \mathrm{L}$ para LT4 e de 0,46 a $-15,4 \mathrm{nmol} / \mathrm{L}$ ou 44 a $1000 \mathrm{ng} / \mathrm{dL}$ para TT3 $3,4,5,9$.

A radiografia de tórax fornece subsídios essenciais para completa avaliação e diagnóstico de doenças ou alterações cardíacas primárias e secundárias, como por exemplo, a dilatação cardíaca secundária ao hipertireoidismo. As radiografias torácicas também podem sugerir o prognóstico e a terapia a ser instituída, servindo como meio de acompanhamento da evolução da doença ${ }^{10}$.

Para Van Den Broek e Darke ${ }^{11}$, a radiografia torácica assume grande valor na avaliação do sistema cardiovascular tanto em cães como em gatos. Em gatos, ao contrário de cães, há menor variação no tamanho e forma do tórax, possibilitando melhor definição das medidas cardíacas.

Segundo Myer e Bonagura ${ }^{12}$, a silhueta cardíaca dos felinos no exame radiográfico tende a ser mais elíptica e alongada quando comparada com os caninos. A área ventricular do coração em ambas as espécies ocupa aproximadamente 2,0 a 2,5 espaços intercostais e a silhueta cardíaca ocupa aproximadamente $70 \%$ da distância do esterno até a coluna espinhal no ponto de maior extensão dorsal do coração em projeção lateral. Em projeções dorsoventral e ventro-dorsal apresenta-se ovalado, parecendo ser um pouco mais delgado e alongado que corações de cães.

No método empírico de avaliação de radiografias torácicas analisa-se o espaço que o coração ocupa no tórax e sua relação com as outras estruturas torácicas. Este método ainda hoje utilizado por radiologistas e cardiologistas, depende muito da experiência do examinador. As desvantagens são a variações do eixo cardíaco, conformações do tórax, fase da respiração, sobreposição das costelas e pontos empíricos de mensuração ${ }^{13}$.

A determinação do tamanho cardíaco é importante na avaliação dos pacientes com doenças cardíacas, visto que o aumento da silhueta cardíaca nas radiografias torácicas é indicativo de alterações cardíacas ${ }^{10}$. Mensurações cardíacas utilizando métodos matemáticos já foram descritas, tanto para cães como em $\operatorname{gatos}^{11,14,15}$. As mensurações apresentam como vantagens a possibilidade de serem utilizadas sem dificuldades por profissionais, facilitando a avaliação da silhueta cardíaca, e diminuindo a subjetividade da avaliação cardíaca $^{10}$.

Buchanan e Bücheler ${ }^{10}$ descreveram a mensuração radiográfica do coração, utilizando o sistema de escala vertebral ou "vertebral scale system" (VHS), em cães e mais recentemente em gatos ${ }^{14}$. O VHS consiste em determinar o valor absoluto e relativo do tamanho cardíaco. Este método consiste em correlacionar o tamanho cardíaco com estruturas esqueléticas como vértebras torácicas e esternebras. Nas mensurações cardíacas há relação das dimensões do coração com o comprimento das vértebras torácicas, portanto, o VHS é uma boa ferramenta na avaliação da progressão da doença cardíaca.

O VHS criou forte expectativa no auxílio do diagnóstico radiográfico de doenças cardíacas, entretanto há poucas publicações dando suporte a esta hipótese ${ }^{16}$. Em 1995, Kim $^{17}$ utilizando o VHS encontrou aumento cardíaco somente em $30 \%$ dos cães com dirofilariose e Melian, Stenacci e Peterson ${ }^{18}$ observaram que somente $27 \%$ dos cães com hipoadrecorticismo tinham valores dentro do normal. Observou-se maior acurácia do VHS em cães que apresentam hipertrofia exêntrica ou dilatação das câmaras cardíacas, em comparação com doenças que resultam em hipertrofia concêntrica com espessamento da parede e diminuição do lúmen ventricular, com pouco aumento das dimensões externas, como a estenose aórtica $^{16}$.

Fase respiratória, ciclo cardíaco, erros de posicionamento e erros na mensuração foram descritos como possíveis fatores na variabilidade na mensuração ${ }^{10}$. Esses fatores 
combinados com doença cardíaca sem cardiomegalia, em cães, podem limitar o VHS como teste para doença cardíaca. É importante ressaltar que o diagnóstico da doença cardíaca não pode ser baseado somente no VHS; é necessário associar sinais clínicos, achados físicos e também exames como ecocardiografia e eletrocardiografia ${ }^{16}$.

Os valores normais do VHS foram descritos por diversos autores ${ }^{14,15,19}$.

O objetivo deste trabalho foi identificar alterações no tamanho cardíaco utilizando o "vertebral heart size" (VHS) em gatos com hipertireoidismo, durante 42 dias.

\section{Materiais e Método}

Foram estudados 19 gatos, adultos jovens, sadios, sem raça definida, com média de peso corporal de 3,25 $\pm 0,67 \mathrm{~kg}$. Os animais foram alojados em jaulas individuais de ferro esmaltado, alimentados com ração seca comercial e água a vontade. Os estudos foram iniciados após um período de 15 dias de adaptação dos gatos ao ambiente e manejo. No início deste período os animais foram vacinados, vermifugados e receberam tratamento com antiparasitário tópico.

A tireotoxicose foi induzida pela administração oral, de comprimidos, de levotiroxina sódica $(150 \mathrm{mg} / \mathrm{kg} /$ dia $)$ durante 42 dias. Administrava-se o hormônio entre as sete e oito horas da manhã. Semanalmente, os gatos eram pesados e os animais que apresentavam diminuição do peso corporal secundário aos efeitos do excesso dos hormônios tireoidianos, as doses de levotiroxina sódica foram recalculadas e ajustadas para a manutenção da dose de 150 $\mathrm{mg} / \mathrm{kg} /$ dia.

Os animais foram avaliados em sete momentos (M). O M0, feito antes da administração da levotiroxina sódica, resultou nos valores basais dos diversos parâmetros estudados. Durante o período experimental, cuja duração foi de 42 dias, foram considerados outros seis momentos de avaliação: aos sete dias (M1), aos 14 dias (M2), aos 21 dias (M3), aos 28 dias (M4), aos 35 dias (M5) e aos 42 dias (M6). A coleta de sangue, para obtenção do soro ou do sangue total, foi realizada quatro horas após a administração da levotiroxina sódica.

A dosagem dos níveis séricos de T'T3, TT4, LT4 foi determinada pela técnica de radioimunoensaio (RIA) em fase sólida, utilizando-se conjunto de reagente comercial (Coat a Count ${ }^{\circledR}$ Total $\mathrm{T}_{3}$, Diagnostic Products Co., Los Angeles, C.A., USA).

O exame radiográfico do tórax seguiu os mesmos momentos das colheitas de sangue. Os gatos foram mantidos por contenção para a execução das radiografias torácicas na projeção lateral (L) direita, dorsoventral (DV) e ventrodorsal (VD).

Para a mensuração do coração utilizou-se o método proposto por Lister e Buchanan. Com o auxílio de paquímetro e compasso foram obtidos, nas projeções lateral direita, DV e VD, os valores em centímetros e depois transformados em valores com unidade de vértebra torácica com aproximação de 0,1 vértebras, medidos a partir da margem cranial da quarta vértebra torácica (T4). Para obtenção do VHS, nas três projeções realizadas, somou-se os valores do eixo curto e longo em vértebras. $\mathrm{Na}$ projeção lateral (Figura 1) as medidas realizadas foram: eixo cardíaco longo lateral (ECLL): do ápice à base cardíaca, na intersecção da bifurcação traqueal. Esta dimensão reflete a combinação do tamanho do átrio e ventrículo esquerdo; eixo cardíaco curto lateral (ECCL): perpendicular à mensuração do eixo longo, utilizando o ponto máximo de largura, comumente este ponto situa-se na intersecção da silhueta cardíaca com a borda ventral da veia cava caudal; soma do eixo cardíaco lateral (SECL) ou VHS equivale a soma das mensurações do ECLL e do ECCL. Nas posições DV (Figura 2) e VD (Figura 3) as medidas realizadas foram: eixo cardíaco longo VD (ECLVD) e DV (ECLDV): distância do ápice à base cardíaca; eixo curto cardíaco VD (ECCVD) e DV (ECCDV): perpendicular à mensuração do ECLVD ou do ECLDV, na largura máxima do coração; 
soma do eixo cardíaco VD (SECVD) ou VHS equivale a soma das mensurações de ECLVD e ECCVD; soma do eixo cardíaco DV (SECDV) ou VHS equivale a soma do ECLDV e ECCDV.

Para o estudo das variáveis mensuradas em momentos consecutivos foi considerada a análise de medidas repetidas. Quando a variável apresentou adequação à distribuição normal de probabilidade, o procedimento adotado foi o paramétrico, enquanto que, na ausência desta optou-se pelo procedimento não paramétrico. $\mathrm{O}$ estudo das associações de algumas variáveis de interesse em relação a TT4 e LT4 foi realizado considerando o coeficiente de correlação linear Spearrman e o nível de significância utilizado foi de $5 \%{ }^{20}$.

\section{Resultados}

As concentrações séricas da TT4, em $\mathrm{nmoL} / \mathrm{L}$, foram 36,88 $\pm 19,85 \mathrm{em} \mathrm{M0}$, 90,03 $\pm 51,88 \mathrm{em} \mathrm{M1,} \mathrm{106,91 \pm 67,01} \mathrm{em} \mathrm{M2,}$ $108,16 \pm 67,57$ em M3, 116,94 $\pm 55,49$ em M4, 104,17 $\pm 66,61$ em M5 e 104,52 $\pm 68,87$ em M6. Observou-se diferença estatisticamente significativa $(\mathrm{P}<0,001)$ entre $\mathrm{M} 0$ e os demais momentos.

A FT4 também apresentou diferença estatisticamente significativa $(\mathrm{P}<0,001)$ entre M0 $(4,55 \pm 3,05 \mathrm{ng} / \mathrm{dL})$ e os demais momentos. Os valores de M1 a M6 foram $15,35 \pm 12,42 \mathrm{ng} / \mathrm{dL}, 14,03 \pm 8,47 \mathrm{ng} / \mathrm{dL}$, $12,91 \pm 9,03 \mathrm{ng} / \mathrm{dL}, 16,54 \pm 13,03 \mathrm{ng} / \mathrm{dL}$, 15,62 ng/dL e 12,51 ng/dL, respectivamente.

A TT3 não apresentou diferença significativa entre os momentos, os valores de M0 a M6, respectivamente foram 1,38 $\pm 2,16 \mathrm{nmoL} / \mathrm{L}, 1,27 \pm 0,73 \mathrm{nmoL} / \mathrm{L}$, $1,21 \pm 0,49 \mathrm{nmoL} / \mathrm{L}, 1,05 \pm 0,50 \mathrm{nmoL} / \mathrm{L}$, $0,88 \pm 0,50 \mathrm{nmoL} / \mathrm{L}, 0,98 \pm 0,56 \mathrm{nmoL} / \mathrm{L}$, e 1,08 $\pm 0,50 \mathrm{nmoL} / \mathrm{L}$.

No ECLL foi observada diferença significativa $(\mathrm{P}<0,001)$ de $\mathrm{M} 0$ com relação aos demais momentos, M1 com relação aos demais momentos e, M2 e M3 com os demais momentos. O ECCL apresentou diferença significativa de M0 com relação aos demais momentos e, do M1 com relação a M4 e M6. O VHS lateral apresentou diferença significativa $(\mathrm{P}<0,001)$ de $\mathrm{M} 0$ e de M1 com relação aos demais momentos; e, M2 e M3 com relação a M4, M5 e M6. Os valores do VHS em decúbito lateral apresentaram correlação negativa de $\mathrm{M} 0 \mathrm{a}$ M3 e positiva de M4 a M6, com relação aos valores TT4 e FT4.

O ECLVD apresentou diferença significativa $(\mathrm{P}<0,001)$ de $\mathrm{M} 0$ com relação aos demais momentos e de M1 com relação aos demais momentos e de M2 com relação a M5 e M6. Os resultados do ECCVD de M0, M1 e M2 apresentaram diferença significativa $(\mathrm{P}<0,001)$ dos demais momentos, assim como M3 e M4 com relação aos demais momentos. O VSH-VD apresentou diferença significativa $(\mathrm{P}<0,001)$ em M0 e M1 em relação a M3, M4, M5, M6; do M2 em relação a M5 e M6. Foi observada correlação positiva entre o VHS e TT4 de M0 a M2 e, negativa de M3 a M6 e, correlação negativa entre o VHS-VD e FT4 em todos os momentos observados.

O ECLDV apresentou diferença significativa $(\mathrm{P}<0,05)$ de $\mathrm{M} 0$ em relação aos outros momentos e do M6 em relação aos outros momentos. Não foi observada diferença significativa entre os momentos no ECLDV. O VHS-DV apresentou diferença significativa $(\mathrm{P}<0,001)$ de $\mathrm{M} 0$ em relação aos demais momentos; do M1, M2 e M3 em relação ao M4, M5 e M6. Observou-se correlação positiva entre o VHS DV e TT4 de M4 à M6, negativa de M0 à M3 e, correlação negativa entre o VHS DV e FT4 de M0 à M3 e positiva de M4 à M6.

\section{Discussão}

O resultado da tireotoxicose experimental em gatos apresenta algumas particularidades importantes que diferem da literatura, ou seja, dos casos espontâneos. Um ponto a ser considerado é a idade dos animais (adultos jovens), pois o hipertireoidismo felino espontâneo acomete particularmente gatos velhos ${ }^{4,8}$. Outro ponto é a indução da tireotoxicose em gatos sem alterações e/ou 
Tabela 1 - Medidas descritivas e resultado do teste estatístico da comparação entre momentos das medidas cardíacas, em gatos ( $\mathrm{n}=19$ ) submetidos à tireotoxicose, Botucatu-SP

\begin{tabular}{|c|c|c|c|c|c|c|c|}
\hline \multirow[b]{2}{*}{ Medidas Descritivas } & \multicolumn{7}{|c|}{ Momentos } \\
\hline & $\mathrm{MO}$ & M1 & M2 & M3 & M4 & M5 & M6 \\
\hline \multicolumn{8}{|l|}{ ECLL $(v)^{1}$} \\
\hline Mediana & $4,35^{\mathrm{a}}$ & $4,50^{\mathrm{b}}$ & $4,70^{c}$ & $4,65^{c}$ & $4,80^{\mathrm{d}}$ & $4,80^{d}$ & $4,85^{d}$ \\
\hline Média & 4,450 & 4,528 & 4,633 & 4,672 & 4,750 & 4,794 & 4,833 \\
\hline Desvio Padrão & 0,34 & 0,33 & 0,31 & 0,27 & 0,33 & 0,28 & 0,26 \\
\hline \multicolumn{8}{|l|}{$\mathrm{ECCL}(\mathrm{v})^{1}$} \\
\hline Mediana & $3,00^{\mathrm{a}}$ & $3,10^{\mathrm{b}}$ & $3,20^{b c}$ & $3,25^{\mathrm{bc}}$ & $3,30^{c}$ & $3,25^{\mathrm{bc}}$ & $3,30^{c}$ \\
\hline Média & 3,094 & 3,161 & 3,267 & 3,294 & 3,283 & 3,311 & 3,35 \\
\hline Desvio Padrão & 0,30 & 0,29 & 0,36 & 0,36 & 0,33 & 0,33 & 0,30 \\
\hline \multicolumn{8}{|l|}{ VHS-L'1 } \\
\hline Mediana & $7,30^{\mathrm{a}}$ & $7,45^{\mathrm{b}}$ & $7,85^{c}$ & $7,90^{c}$ & $8,20^{\mathrm{d}}$ & $8,15^{d}$ & $8,20^{f}$ \\
\hline Média & 7,544 & 7,689 & 7,900 & 7,956 & 8,028 & 8,10 & 8,183 \\
\hline Desvio Padrão & 0,53 & 0,55 & 0,56 & 0,46 & 0,47 & 0,51 & 0,46 \\
\hline \multicolumn{8}{|l|}{$\operatorname{ECLVD}(v)^{1}$} \\
\hline Mediana & $4,50^{\mathrm{a}}$ & $4,60^{b}$ & $4,70^{c}$ & $4,80^{\text {cd }}$ & $4,80^{\text {cd }}$ & $4,85^{d}$ & $4,85^{d}$ \\
\hline Média & 4,54 & 4,66 & 4,71 & 4,78 & 4,74 & 4,82 & 4,82 \\
\hline Desvio Padrão & 0,35 & 0,31 & 0,32 & 0,29 & 0,29 & 0,27 & 0,31 \\
\hline \multicolumn{8}{|l|}{$\operatorname{ECCVD}(v)^{1}$} \\
\hline Mediana & $3,25^{\mathrm{a}}$ & $3,30^{\mathrm{a}}$ & $3,30^{\mathrm{a}}$ & $3,35^{\mathrm{b}}$ & $3,45^{\mathrm{c}}$ & $3,50^{\mathrm{d}}$ & $3,55^{d}$ \\
\hline Média & 3,33 & 3,33 & 3,36 & 3,44 & 3,48 & 3,54 & 3,57 \\
\hline Desvio Padrão & 0,28 & 0,21 & 0,22 & 0,30 & 0,28 & 0,25 & 0,25 \\
\hline \multicolumn{8}{|l|}{ VHS-VD ${ }^{1}$} \\
\hline Mediana & $7,75^{\mathrm{a}}$ & $7,80^{\mathrm{a}}$ & $8,05^{\mathrm{ab}}$ & $8,20^{b c}$ & $8,30^{b c}$ & $8,40^{c}$ & $8,45^{c}$ \\
\hline Média & 7,97 & 7,99 & 8,04 & 8,22 & 8,23 & 8,36 & 8,37 \\
\hline Desvio Padrão & 0,57 & 0,43 & 0,45 & 0,49 & 0,49 & 0,43 & 0,48 \\
\hline \multicolumn{8}{|l|}{$\operatorname{ECLDV}(v)^{1}$} \\
\hline Mediana & $4,60^{\mathrm{a}}$ & $4,80^{b}$ & $4,75^{\mathrm{b}}$ & $4,70^{b}$ & $4,80^{\mathrm{b}}$ & $4,80^{b}$ & $4,90^{c}$ \\
\hline Média & 4,56 & 4,67 & 4,68 & 4,68 & 4,76 & 4,80 & 4,84 \\
\hline Desvio Padrão & 0,31 & 0,27 & 0,24 & 0,27 & 0,29 & 0,30 & 0,30 \\
\hline \multicolumn{8}{|l|}{$\operatorname{ECLDV}(v)^{2}$} \\
\hline Mediana & 3,35 & 3,55 & 3,45 & 3,55 & 3,55 & 3,60 & 3,55 \\
\hline Média & 3,47 & 3,51 & 3,49 & 3,55 & 3,56 & 3,59 & 3,59 \\
\hline Desvio Padrão & 0,31 & 0,31 & 0,32 & 0,28 & 0,24 & 0,22 & 0,25 \\
\hline \multicolumn{8}{|l|}{ VHS-DV ${ }^{1}$} \\
\hline Mediana & $7,95^{\mathrm{a}}$ & $8,15^{\mathrm{b}}$ & $8,15^{\mathrm{b}}$ & $8,20^{b}$ & $8,40^{c}$ & $8,45^{c}$ & $8,50^{c}$ \\
\hline Média & 8,04 & 8,19 & 8,17 & 8,23 & 8,32 & 8,39 & 8,43 \\
\hline Desvio Padrão & 0,58 & 0,52 & 0,48 & 0,50 & 0,47 & 0,44 & 0,47 \\
\hline & \multicolumn{7}{|c|}{$1-\mathrm{P}<0,001 ; 2-\mathrm{P}<0,05$} \\
\hline
\end{tabular}




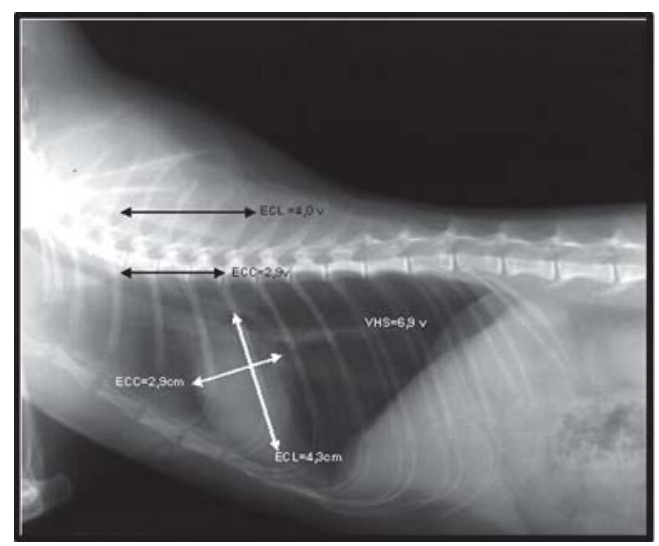

Figura 1 - Radiografia lateral obtida de um gato com hipertireoidismo durante a inspiração. Eixo cardíaco curto (ECC) e longo (ECL)em centímetros (cm) evértebras (v) eo VHS. Botucatu, SP

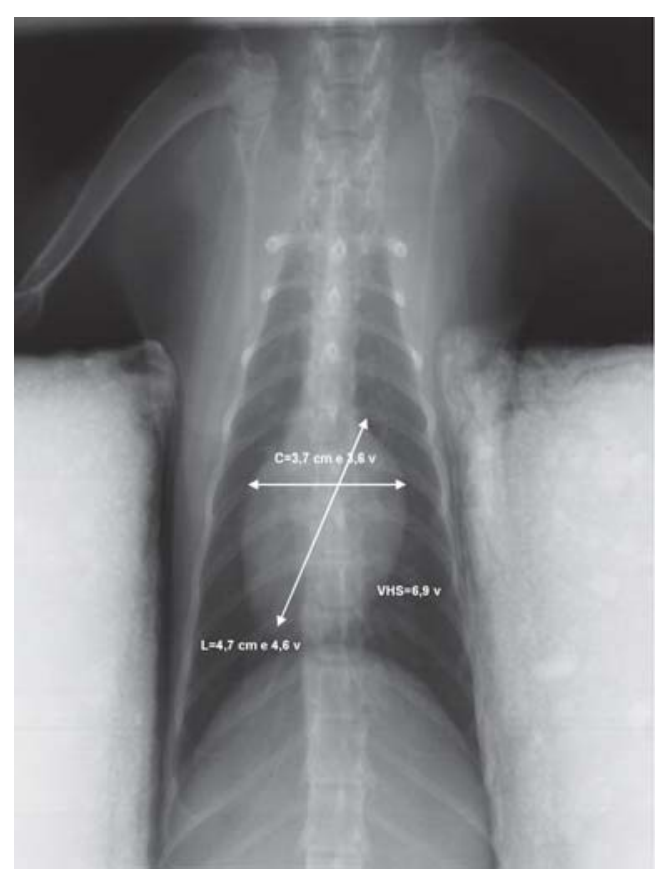

Figura 2- Radiografia ventro-dorsal obtida de um gato com hipertireoidismo durante a inspiração. Eixo cardíaco curto (ECC) e longo (ECL) em centímetros (cm) e vértebras (v) eo VHS. Botucatu, SP

doenças concomitantes, visto que no hipertireoidismo espontâneo os animais são apresentados com doenças não tireoidianas que podem mascarar os resultados do hipertireoidismo, tanto aumentando como diminuindo os valores laboratoriais.

As concentrações séricas da TT4, da LT4 e da TT3 em M0 situaram-se dentro

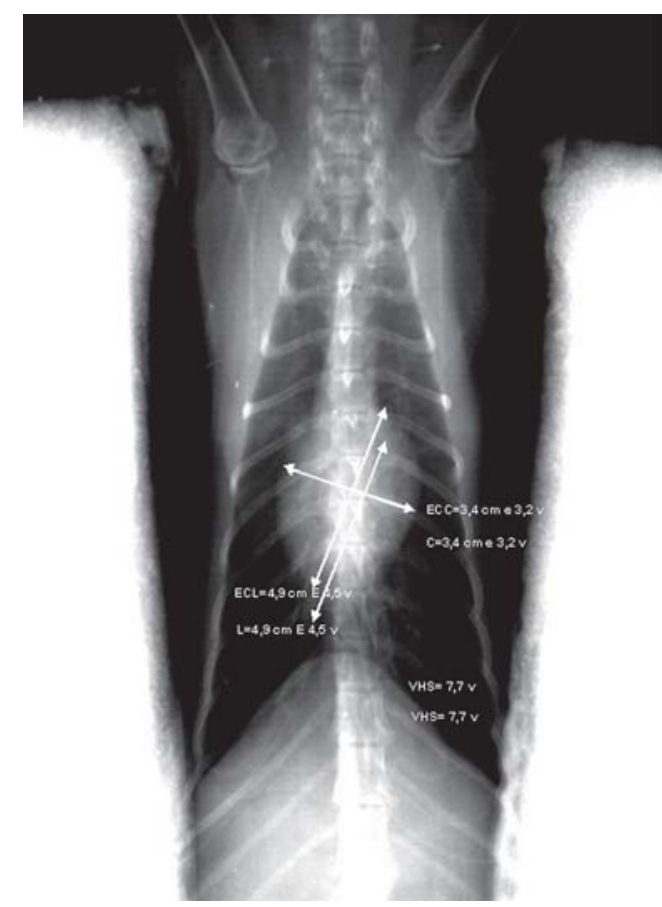

Figura 3- Radiografia ventro-dorsal obtida de um gato com hipertireoidismo durante a inspiração. Eixo cardíaco curto (ECC) e longo (ECL) em centímetros (cm) e vértebras (v) eo VHS. Botucatu, SP

dos valores de referência para gatos ${ }^{4,5,9}$, bem como as concentrações de M1 à M6 são compatíveis com os do hipertireoidismo espontâneo ${ }^{3,9}$. Isso demonstra que o protocolo experimental foi adequado para induzir tireotoxicose nos gatos e provocar alterações no VHS.

O VHS nas três projeções apresentou um aumento progressivo com diferença estatística significativa dos momentos finais em relação aos momentos iniciais. Foi observada correlação negativa entre o VHS lateral e os hormônios tireoidianos, isso pode indicar que os hormônios tireoidianos exerceram pouca influência na progressão da doença cardíaca. O tempo de experimento, 42 dias, pode ser o fator determinante.

O aumento com diferença significativa entre o M0 e os demais momentos tanto na mensuração do eixo cardíaco curto como do eixo cardíaco longo, indicam que houve aumento tanto de átrios como de ventrículos. 
Os valores encontrados na incidência lateral, VD e DV no MO são semelhantes aos descritos por Costa et al. ${ }^{19}$, mas o eixo cardíaco curto e longo e conseqüentemente o VHS nas três projeções foram menores que o descrito por Lister e Buchanan ${ }^{14}$. A partir do M1 os gatos começaram a perder peso e, portanto deve-se lembrar que a gordura pericárdica influi no VHS ${ }^{15}$. O VHSlateral demonstrou aumento significativo nos momentos finais quando comparado com os primeiros momentos. O VHS-L no M1 foi semelhante ao valor normal $(7,5 \pm 0,3$ vértebras) para felinos, o VHS foi maior que este valor a partir de M2. Estes resultados sugerem que o VHS-L é um bom método para avaliação da progressão da doença cardíaca em gatos.

O VHS-DV e VD tiveram aumentos progressivos e os momentos finais apresentaram diferença significativa em relação aos momentos iniciais. Resultado semelhante havia sido descrito por Myer e Bonagura $^{12}$, na observação empírica. A variação do VHS-DV foi menor quando comparada ao VHS lateral e VD. O ECCDV não apresentou diferença significativa entre os momentos e estava dentro dos limites considerado normais para felinos. O VHSVD foi ligeiramente superior aos valores descritos por Lister e Buchanan ${ }^{14}$ somente nos momentos finais.

A correlação positiva do VHS lateral e dorso-ventral em relação à T'T4 e FT4 nos momentos finais sugerem que o aumento crônico dos hormônios tireoidianos e não o aumento agudo destes hormônios tireoidianos provoca o aumento do coração. A correlação negativa entre VHS-VD e FT4 em todos os momentos e, com a TT4 nos momentos finais, também sugerem que o período de permanência no estado hipertireóideo também possui influência sobre o aumento do VHS e não somente as elevadas concentrações séricas dos hormônios tireoidianos.

Os valores do VHS nas três projeções, mas principalmente na projeção lateral, demonstrou a progressão da doença cardíaca nos animais utilizados neste experimento. Lamb et al. ${ }^{16}$ também observaram uma maior acurácia do VHS em cães com doenças cardíacas que causam hipertrofia concêntrica ou dilatação das câmaras cardíacas, como ocorre no hipertireoidismo. Há descrições radiográficas que relatam a presença de cardiomegalia leve a grave, bem como derrame pleural e edema pulmonar, ${ }^{5,6,7}$. A diferença entre o VHS e estas descrições é que as primeiras são medidas quantitativas e as segundas qualitativas e empíricas, as últimas sofrem maior influência pessoal do que as primeiras. Com a utilização do VHS pode-se observar uma cardiomegalia discreta provocada pela tireotoxicose e acompanhar a progressão ou remissão dos achados após a instituição da terapia.

\section{Conclusões}

O VHS demonstrou ser um método eficiente para mensuração cardíaca e na detecção da doença cardíaca progressiva em gatos com hipertireoidismo experimental. Este método é prático, fácil e barato e trouxe bons resultados no acompanhamento da doença cardíaca. A projeção lateral foi mais eficiente que as projeções DV e VD na avaliação cardíaca, quando se utiliza o VHS para mensuração cardíaca em gatos com tireotoxicose experimental.

Conclui-se que a progressão da doença cardíaca tireotóxica provoca aumento do VHS lentamente progressivo, este aumento aparentemente relaciona-se à cronicidade $\mathrm{da}$ doença e não às concentrações séricas dos hormônios tireoidianos. Sugere-se que o período de evolução do hipertireoidismo está relacionado ao aumento do VHS e não somente as concentrações séricas dos hormônios.

O VHS é um dado complementar para a avaliação radiográfica, não devendo ser utilizado como único parâmetro. Permite a caracterização de cardiomegalia e também avaliação de exames seriados no acompanhamento do paciente.

\section{Agradecimentos}

Os autores agradecem à FAPESP pelo financiamento da bolsa de mestrado 
que possibilitou esta pesquisa e os técnicos do Laboratório de Radiologia da FMVZ-UNESP.

\section{Vertebral scale system to measure heart size in radiographs in experimentally hyperthyroidism in the cats}

\section{Abstract}

Feline hyperthyroidism (thyrotoxicosis) is now recognised as the most common endocrine disease of the domestic cat. Feline hyperthyroidism cause multisystemic disorder associated with incresead circulating concentrations of the thyroid hormones, tri-iodothyronine (T3) and thyroxine (T4). Cardiovascular abnormalities in cats with spontaneous hyperthyroid have been described. Objective to determine heart size of progressing experimental thyrotoxicosis of 19 cats, using a vertebral scale system (VHS). The lateral, dorsoventral e ventrodorsal radiographs thoracic were measured using a vertebral scale system. Lateral radiographs thoracic to efficient develop that DV and VD. The VHS method is easy to use, allows objective assesment of heart size in cats hyperthyroid, and may be helpful to identify cardiomegaly and heart size progressing.

\section{Referências}

1 MOONEY, C. T. Feline hyperthyroidism: diagnosis and therapeutics. Veterinary Clinical North American Small Animal Practice, v. 31, n. 5, v. 963-983, 2001.

2 MOONEY, C. T; . THODAY, K.L.; DOXEY, D. L. Serum thyroxine and triidothyronine responses of hyperthyroid cats to thyrotropin. American Journal Veterinary Research, v. 57, n. 7, p. 987-991, 1996.

3 MOONEY, C. T.; LITTLE, C. J. L.; MACRAE, A. W. Effect of illness not associated with the thyroid gland on serum total and free thyroxine concentrations in cats. Journal American Veterinary Medical Association, v. 208, n. 12, p. 2004-2008, 1996.

4 PETERSON, M A · MELIÁN C * NICHOLS, R. Measurement of serum concentrations of free thyroxine, total thyroxine, and total triiodothyronine in cats with hyperthyroidism and cats with nonthyroidal disease. Journal American Veterinary Medical Association, v 218, p. 529-536, 2001.

5 PETERSON, M. E. et al. Feline hyperthyroidism: Pretreatment clinical and laboratory evaluation of 131 cases. Journal American Veterinary Medical Association, v. 183, n. 1, p. 103-110, 1983.

6 JACOBS, G.; HUTSIN, C.; DOUGHERTY, P. Congestive heart failure associated with hyperthyroidism in cats. Journal American Veterinary Medical Association, v.188, p. 52-56, 1986.

7 FOX, P. R.; PETERSON, M. E.; BROUSSARD, J. D. Eletrocardiographic and radiographic changes in cats with hyperthyroidim: comparison of populations evaluated during 1992-1993 vs 1979-1982. Journal American Animal Hospital Association, v. 35, n. 1, p.
27-31, 1999.

8 THODAY, K. L.; MOONEY, C. T. Historical clinical and laboratory features of 126 hyperthyroid cats. Veterinary Record, v. 131, n. 12, p. 257-64, 1992.

9 FELDMAN, E. C.; NELSON, R. W. Feline hyperthyroidism (thyrotoxicosis).In: FELDMAN, E. C. NELSON, R. W. Canine and feline endocrinology and reproduction. Philadelphia: W.B. Saunders, 1996. p. 118-166.

10 BUCHANAN, J. W · BÜCHELER, J. B. Vertebral scale system to measure canine heart size in radiographs. Journal American Veterinary Medical Association, v. 206, n. 2, p. 194-199, 1995.

11 VAN DEN BROEK, A. H. M. DARKE, P. G. G. Cardiac mensuraments on thoracic radiographs of cats. Journal Small Animal Practice, v. 28, p. 125-135, 1987.

12 MYER, C. W.; BONAGURA, J. A. Survey radiography of the heart. Veterinary Clinical North American Small Animal Practice, v. 12, p. 213-217, 1982.

13 BUCHANAN, I. W Vertebral scale system to measure heart size in radiographs. Veterinary Clinical North American Small Animal Practice, v. 30, n. 2, p. 379-393, 2000

14 LISTER, A. L.; BUCHANAN, J. W. Radiographic and echocardiographic measurement of the heart in obeses cats. Veterinary Radiology Ultrasound, v. 41, n. 4 , p. $320-325,2000$

15 LISTER, A. L.; BUCHANAN, J. W. Vertebral scale system to measure heart size in radiographs of cats. Journal American Veterinary Medical Association, v. 216 , n. 2, p. 210-214, 2000 
$16 \mathrm{LAMB}, \mathrm{C}$. R. et al. Assessment of the value of the vertebral heart scale in the radiographic diagnosis of cardiac disease in dogs. Veterinary Record, v. 146, n. 24, p. 687-90, 2000.

17 KIM, R. Radiographic evaluation of canine heartworn disease. Korean Journal Veterinary Clinical Medicine, v. 12, p. 265-275, 1995.

18 MÉLIAN, C.; STEFANACCI, J.; PETERSON, M. E. Radiographic findings in dogs with naturally-occcurring primary hypoadrenocorticism. Journal American Animal Hospital Association, v. 35, n. 3, p. 208-212, 1999.

19 COSTA, F. S. et al. Medidas radiográficas absolutas e relativas do coração em gatos normais (Felis catus, L. 1758). In: CONGRESSO INTERNACIONAL DE MEDICINA FELINA, 2., 2001. Rio de Janeiro, Anais... Rio de Janeiro, 2001.

20 STREINER, D. L.; NORMAN, G. R. Bioestatistics: the bare essentials. St. Louis: Mosby - Year Book, 1994. $260 \mathrm{p}$. 